\title{
Chinese herbal medicine for the treatment of chronic rhinosinusitis: protocol for a systematic review and meta-analysis
}

\section{Shouliang Hu}

Chengdu University of Traditional Chinese Medicine https://orcid.org/0000-0001-9416-7407

\section{Lu Li}

Chengdu University of Traditional Chinese Medicine

\section{Xin Zhu}

Chengdu University of Traditional Chinese Medicine

\section{Yijie Fu}

Chengdu University

Hui Li ( $\nabla$ ttlihui@163.com )

Chengdu University

\section{Tianmin Zhu}

Chengdu University of Traditional Chinese Medicine

\section{Protocol}

Keywords: Chronic rhinosinusitis, Chinese herbal medicine, Systematic review, Meta-analysis

Posted Date: May 23rd, 2020

DOl: https://doi.org/10.21203/rs.3.rs-28493/v1

License: (1) This work is licensed under a Creative Commons Attribution 4.0 International License. Read Full License 


\section{Abstract}

Background:

In east Asian countries, the traditional treatment is to treat chronic rhinosinusitis(CRS) with some herbal formulas. However, there is a lack of clinical evidence to support the effect of the drug. Here, we describe a randomized controlled trial to investigate the efficacy and safety of herbal formulations for the treatment of CRS.

Methods:

We will search electronic databases including the Cochrane Central Register of Controlled Trials (CENTRAL), Chinese Biomedical Literature Database (CBM), PubMed, Embase, Chinese Science and Technology Periodical Database (VIP), China National Knowledge Infrastructure (CNKI), and Wanfang database using keywords related to CRS and Chinese herbal medicine. Extensive database search was carried out for CRS related randomized controlled trials. Two reviewers(SH and LL) will independently filter the search results to determine eligible articles, complete data collection.The quality of individual trials will be assessed by (XZ and YF) using the Cochrane collaborative bias risk assessment tool. We will calculate the relative risk and $95 \%$ confidence interval $(\mathrm{Cl})$ of the dichotomy results and the weighted average difference, as well as the corresponding $95 \%$ confidence interval of the continuous results. Statistical methods such as subgroup analysis and sensitivity analysis will be used to investigate the sources of heterogeneity.

Discussion:

The systematic review and meta-analysis will provide evidence for the quality of life and the safety of traditional Chinese medicine in CRS patients. This study will provide a high quality research method for the therapeutic effect of Chinese herbal medicine on CRS, and will also help broaden research horizons for the complementary and alternative therapy of CRS.

Systematic review registration

PROSPERO registration number: CRD42019123047

(https://www.crd.york.ac.uk/prospero/display_record.php?ID=CRD42019123047).

\section{Background}

CRS is an inflammatory reactive disease of nasal mucosa. The main symptoms inlude nasal obstruction, runny nose, pain or swelling of head and face. With the aggravation of the disease, there may be loss of smell, memory and attention [1].The above symptoms lasted for more than 12 weeks, with 2 or more than 2 symptoms, which can be used for diagnosis according to the toEPOS 2012 standard[2].CRS can occur in all ages with a incidence of $5 \%-15 \%[3]$. The long-term recurrence of CRS not only seriously affects the 
quality of life of patients, but also greatly increases the social medical costs. According to relevant research reports, the annual medical expenses of CRS treatment in the United States exceed 22 billion US dollars, which brings huge economic pressure to the medical system in the United States[4].At present, the drug treatment of CRS mainly includes antibiotics, antihistamines or corticosteroids, and so on[2] .Long term use of these drugs has obvious side effects, which makes the current treatment effect is not well.In order to seek better therapeutic effect, people are speeding up the exploration and research of related alternative drugs or adjuvant therapy. For example, the treatment of CRS with Chinese herbal medicine has been gradually studied by more and more people.

Chinese herbal medicine has a long history in the treatment of CRS. Xanthium powder, which comes from Ji Sheng Fang of song dynasty, is the basic and classic prescription for treating nasitis.Randomized controlled trials showed that the Chinese herbal medicine compound Xanthium powder and Houttuynia cordata were similar to macrolide in the treatment of CRS. At the same time, this study also found that Gegen Decoction and Longdan Xiegan decoction had antiviral effect on torhinolaryngologic diseases[56].It was confirmed that[7] herbal dry extract BNO 1011 could increase the clearance rate of mucociliary by up regulating the culture of nasal cavity in vitro and $\mathrm{Cl}$ - secretion of mouse epithelial cells in vivo. Chinese herbal medicine has the functions of dissolving viscosity, antiviral, antibacterial, antiinflammatory and promoting secretion. Plant therapy has high tolerance and safety in the treatment of CRS.[8]With the application of Chinese herbal medicine in CRS, there is no unified conclusion on the therapeutic effect of Chinese herbal medicine. This paper will systematically review and analyze the efficacy and safety of Chinese herbal medicine in CRS.

\section{Methods/design}

\section{Study registration}

The methodology to perform this systematic review was developed according to recommendations from the Preferred Reporting Items for Systematic Reviews and Meta-Analyses Protocols (PRISMA-P) statement [9]. The corresponding Checklist of PRISMA-P can be found in Additional file 1. This protocol is currently registered in the International Prospective Register of Systematic Reviews (PROSPERO): CRD42019123047.

\section{Eligibility criteria}

\section{Inclusion criteria}

Types of studies Randomized controlled trials will be included. Chinese herbal medicine does not include combination drugs with western medicine, etc. There are no restrictions on blinding, follow-up or publishing status. Both Chinese and English publications are available. 
Types of participants According to the European guidelines for the classification of CRS, CRS can be divided into patients with nasal polyps (CRSwNP) or patients without nasal polyps (CRSsNP)[3]. Inclusion criteria for CRS need to meet EPOS 2012 definition specifications[2].Among them, some patients with cystic fibrosis, myxosinusitis, malignant polyps, eosinophilic fungal sinusitis, allergic fungal sinusitis, inverted papilloma and primary ciliary dyskinesia will be excluded.

Types of interventions Chinese herbal medicines, including herbal extracts, single or mixed herbal formulas, regardless of composition or form. Chinese herbal medicines combined with one or more other drug interventions will also be included. There is no limit to the dosage, frequency, duration or follow-up time of treatment.

Type of comparators There will be no restrictions with respect to the type of comparator. The comparators are likely to include western medicine, placebo, acupuncture, surgical treatment and other treatments.

Type of outcome measurements Our primary outcomes include main symptom efficacy score (including nasal obstruction, runny nose, sneezing, dizziness and pain et al), treatment-related adverse events and health-related quality of life scores(such as the SinoNasal Outcome Tes-22(SNOT-22) or SNOT-20). Secondary outcomes include total nasal symptom score,recurrence rate, endoscopic score (depending on the population, either nasalpolyps size score, e.g. Lund-Kennedy), computerised tomography(CT) scan scores (e.g.Lund-Mackay) and Visual Analogue Score(VAS).If other results are reported in a qualified study, they will be extracted and reported, but when we use any of these results in our review, special attention will be paid to the possibility of selectively reporting deviations.

\section{Exclusion criteria}

The exclusion criteria were as follows: (1) it was not a randomized controlled trial; (2) it could not obtain complete data after repeated literature or contact with the original author; (3) it was not an original literature.

\section{Databases and search strategy}

The systematic search will be conducted with English and Chinese. The database for electronic retrieval includes: Cochrane Central Register of Controlled Trials (CENTRAL), Chinese Biomedical Literature Database (CBM), PubMed, Embase, Chinese Science and Technology Periodical Database (VIP), China National Knowledge Infrastructure (CNKI), and Wanfang database (Wanfang Data). All databases will be searched from the build database to May 6, 2019 using a predetermined search strategy.The full search strategy for PubMed is provided in Additional file 2, and similar strategies will be applied to other electronic databases. A reference list of relevant tests and reviews will be searched. Related conference papers, journal references and magazines without electronic version will be manually searched to identify 
all gray literatures. The search words will be modified to adapt to different databases.Furthermore, potentially relevant studies will be identified by manual search of reference list of included trials and review articles.

\section{Study selection}

The two reviewers (SH and $\mathrm{LL}$ ) will screen the titles and abstracts retrieved from the database with unified standards one by one to check whether the required qualified tests are included. If the filter topic or summary is related to the review topic, they will be evaluated in full one by one. In the screening process, if there are differences, we will focus on the inclusion and exclusion criteria, or the third author (XZ) will decide.Dtails of the entire selection procedure will be shown in a flow chart (see figure 1).

\section{Data extraction and management}

The two reviewers (SH and LL) will collect data through standardized template format, extract data one by one from the included experiments, and resolve disputes through consultation or discussion with the third author $(\mathrm{XZ})$. If the data and information required in the article are incomplete, we will contact the author of the experiment to complete the required information.

We will extract the following data from each included trial: the basic characteristics of the study literature (author, year of publication and journal), the trial design method (including random method, assignment concealment or not), the patient characteristics (total sample size, average age, gender, race, course of disease, eligibility criteria), the experimental group and the control group (intervention, number of patients in each group, number of shedding), follow-up time, adverse reactions).

\section{Dealing with missing data}

When the data is lost or the information is incomplete, two independent reviewers(SH and LL)will contact the relevant author by email or phone tracking to obtain the missing data. If there is a lack of data in the end, we will analyze the data based on the principle of intention to treat (ITT)[10]. We can use standard error, precise P-value or $95 \%$ confidence interval $(\mathrm{Cl})$ to approximate the standard deviation[11].Considering that there may be differences in the quality of medical literature, sensitivity analysis will be conducted for relevant data if necessary.

\section{Assessment of risk of bias in included studies}

Cochrane's "bias risk" assessment tool [12] will be used to assess bias risk in randomized controlled trials. The bias assessment of the randomized controlled trials included mainly includes the following aspects:Selection bias (e.g. method generated by random assignment sequence and assignment 
concealment), implementation bias (e.g. double blind for participants and implementers), measurement bias (e.g. blind for result evaluation), loss bias (e.g. reasonable analysis for loss data), publication bias (e.g. existence of selective report) and others Source bias. All the included test biases are divided into three grades of "low", "high" and "unclear", which shall be completed or noted according to the specific situation. Two reviewers(XZ and YF) will evaluate independently and any differences will be resolved through discussion or by a third author (SH).

\section{Data synthesis}

Statistical analyses will be performed using RevMan 5.3.5 software (The Cochrane Collaboration).Pooled risk ratio (RR) with $95 \%$ confidence interval $(\mathrm{Cl})$ of dichotomous outcomes will be used to report effect estimates.Continuous data will be presented as mean difference (MD) with $95 \% \mathrm{Cl}$. If different measurement scales are used, standardized mean difference (SMD) will be performed to analyze continuous data. The heterogeneity of different experimental studies was analyzed by Chi-square test and I2 statistics[13]. According to Cochrane system evaluation, when the P-value of the Chi-square test is greater than 0.10 or $12<50 \%$, the heterogeneity difference of related research is acceptable.If studies included are homogeneous in the field of extracted information, fixed-effect model will be used. When the P-value of the Chi-square test in this study is $\leq 0.10$ or $12 \geq 50 \%$, it is considered that the statistical heterogeneity is high, and the collected literature is further screened for quality. In order to find out the source of heterogeneity in experimental study, sensitivity analysis, subgroup analysis or meta regression analysis will be carried out on the basis of age, course of disease, treatment time, CRS type, dose difference and other factors. For the research with high heterogeneity, the random effect model can be used to evaluate the effect amount.

\section{Discussion}

Hormones, sterols and other drugs are commonly used in the treatment of CRS, which are criticized for their obvious side effects[14]. In order to optimize the current treatment plan and means, we need to find more effective treatment drugs or alternative complementary therapy.In a systematic review of four randomized controlled trials, no serious reactions occurred during the treatment of CRS with herbal medicine, but further certification is needed due to the quality and quantity limitations of inclusion[15].Traditional Chinese medicine has obvious effect on cytokines, especially in improving inflammatory response, enhancing immune function, promoting cilia activation and regulating tissue remodeling, which can reduce congestion and edema of nasal mucosa, promote the transmission of mucosal cilia, and restore the physiological function of nasal mucosa[16].It has been studied that BiYuanshu Oral Liquid can reduce the content of COX-2 and MMP-2 in the nasal mucosa, inhibit the chronic inflammatory response of CRS, and thus have repair function and anti-inflammatory effect on the local nasal mucosa[17].It also has a certain role in reducing postoperative mucosal edema and promoting the recovery of postoperative mucosal inflammation[18]. 
Chinese herbal medicines, including proprietary Chinese medicine, decoction and extract, are commonly used in the treatment of CRS and are widely used.But at present, the therapeutic effect of Chinese herbal medicine on CRS is controversial. The therapeutic effect of Chinese herbal medicine still needs to be certified, and whether it can be included in the treatment of CRS as an alternative therapy is not clear.This study will explore the efficacy and safety of Chinese herbal medicine in the treatment of CRS, so as to draw the corresponding conclusions, and provide the relevant basis for the direction research of CRS replacement therapy. The conclusion of this study will contribute to the pioneering research of CRS treatment scheme and optimize the treatment scheme for clinicians. At the same time, it also helps to reduce the suffering of CRS patients and the cost of the national medical system.

\section{Abbreviations}

CRS Chronic rhinosinusitis

CRSwNP with nasal polyps

CRSsNP without nasal polyps

RCT(s) Randomized controlled trials

$\mathrm{Cl} \quad$ Confidence interval

CT Computerized tomography

ITT Intention to treat

CBM Chinese Biomedical Literature Database

CENTRAL Cochrane Central Register of Controlled Trials

CNKI China National Knowledge Infrastructure

PROSPERO Prospective Register of Systematic Reviews

VIP Chinese Science and Technology Periodical Database

\section{Declarations}

\section{Acknowledgements}

Not applicable.

\section{Author contributions}


Hui Li, Tianmin Zhu designed the study. Shouliang Hu, Lu Li, Xin Zhu, Yijie Fu drafted the manuscript. All authors approved the final manuscript.

\section{Funding}

This work was supported by the National Natural Science Foundation of China (grant number 81674037) and Supported by Sichuan Science and Technology Program (grant number 2018SZ0375).

\section{Availability of data and materials}

Not applicable.

\section{Ethics approval and consent to participate}

Not applicable.

\section{Consent for publication}

Not applicable.

\section{Competing interests}

The authors declare that they have no competing interests.

\section{Footnotes}

\section{Publisher's Note}

Springer Nature remains neutral with regard to jurisdictional claims in published maps and institutional affiliations.

Shouliang Hu and Lu Li contributed equally to this work.

\section{Contributor Information}

Hui Li, E-mail: ttlihui@163.com

Tianmin Zhu, E-mail: tianminzhu@cdutcm.edu.cn 


\section{References}

1.Fokkens WJ, Lund VJ, Mullol J, Claus Bachert C,Alobid I, Baroody F,et al.European Position Paper on Rhinosinusitis and Nasal Polyps 2012.Rhinol Suppl 2012;3:1-298.

2.Fokkens WJ, Lund VJ, Mullol J, Bachert C, Alobid I, Baroody F,et al. EPOS 2012: European position paper on rhinosinusitis and nasal polyps 2012.A summary for otorhinolaryngologists. Rhinology 2012;50:1-12.

3.CAIN R B, LAL D.Update on the management of chronic rhinosinusitis. Infect Drug Resist 2013;6:1-14.

4.Smith KA, Orlandi RR, Rudmik L. Cost of adult chronic rhinosinusitis: a systematic

review. Laryngoscope 2015;125:1547-56.

5.Cheng HY, Huang HH, Yang CM, Lin LT, Lin CC. The in vitro anti-herpes simplex virus type-1 and type-2 activity of Long Dan Xie Gan Tan, a prescription of traditional Chinese medicine. Chemotherapy 2008;54:77-83.

6.Anushiravani M, Bakhshaee M, Taghipour A, Baghdar H, Farshchi MK, Hoseini SS,et al.A systematic review of randomized controlled trials with herbal medicine on chronic rhinosinusitis.Phytother Res 2018 ;32(3):395-401.

7.Cho DY, Skinner D, Mackey C, Lampkin HB, Elder JB, Lim DJ, et al.Herbal dry extract BNO 1011 improves clinical and mucociliary parameters in a rabbit model of chronic rhinosinusitis. Int Forum Allergy Rhinol 2019;9(6):629-637.

8.Passali D, Cambi J, Passali FM, Bellussi LM. Phytoneering: a new way of therapy for rhinosinusitis. Acta Otorhinolaryngol Ital 2015;35(1):1-8.

9.Shamseer L, Moher D, Clarke M, Ghersi D, Liberati A, Petticrew M,et al.Preferred reporting items for systematic review and meta-analysis protocols (PRISMA-P) 2015: elaboration and explanation. BMJ. 2015;349(1):g7647.

10.Nüesch E, Trelle S, Reichenbach S, Rutjes AWS, Bürgi E, Scherer M, et al. The effects of excluding patients from the analysis in randomised controlled trials: metaepidemiological study.BMJ 2009;339:b3244.

11.The Nordic Cochrane Centre, The Cochrane Collaboration. Review Manager (RevMan). Version 5.3. Copenhagen: The Nordic Cochrane Centre, The Cochrane Collaboration, 2014.

12.Higgins JPT, Green S, eds. Cochrane Handbook for Systematic Reviews of Interventions Version 5.1.0 [updated March 2011].The Cochrane Collaboration, 2011. Available at: www.cochrane-handbook.org.The Cochrane Collaboration. Assecced December 28, 2018. 
13.Higgins JPT, Altman DG, Gøtzsche PC, Jüni P, Moher D, Oxman AD, et al. The Cochrane Collaboration's tool for assessing risk of bias in randomized trials. Br Med J 2011;343:1-9.

14.Kwon E, O'Rourke MC. Chronic Sinusitis. In: StatPearls. Treasure Island (FL): StatPearls Publishing; 2020.

15.Son MJ, Kwon O, Kim S, Kim YN, Jung SY, Kim BY,et al.Safety and efficacy of Galgeun-tang-gacheongung-sinyi, a herbal formula, for the treatment of chronic rhinosinusitis: A study protocol for a randomized controlled trial. Medicine (Baltimore)2018;97(25):e11196.

16.Huang CC,Wang H,Jia ZQ. Research progress on treatment of sinusitis with traditional Chinese medicine and its influence on related cytokines. Hebei Traditional Chinese medicine 2019,41 (03): 476480 .

17.Linlin Yang. Study on the effect of Biyuanshu Oral Liquid on the expression of COX-2 and MMP-2 in chronic sinusitis. Master Thesis. Chengdu Uniersity of Traditional Chinese Medicine, Chengdu, Sichuan, China 2014.

18.Zhang DZ, Liu M, Zhang QX, Li XQ, Xian KL, Li JL, et al. Systematic evaluation of the effect of traditional Chinese medicine irrigation on the postoperative cavity recovery of chronic sinusitis after endoscopic sinus surgery [J]. Chinese Journal of evidence based medicine, 2011,11 (05): 576-590.

\section{Figures}




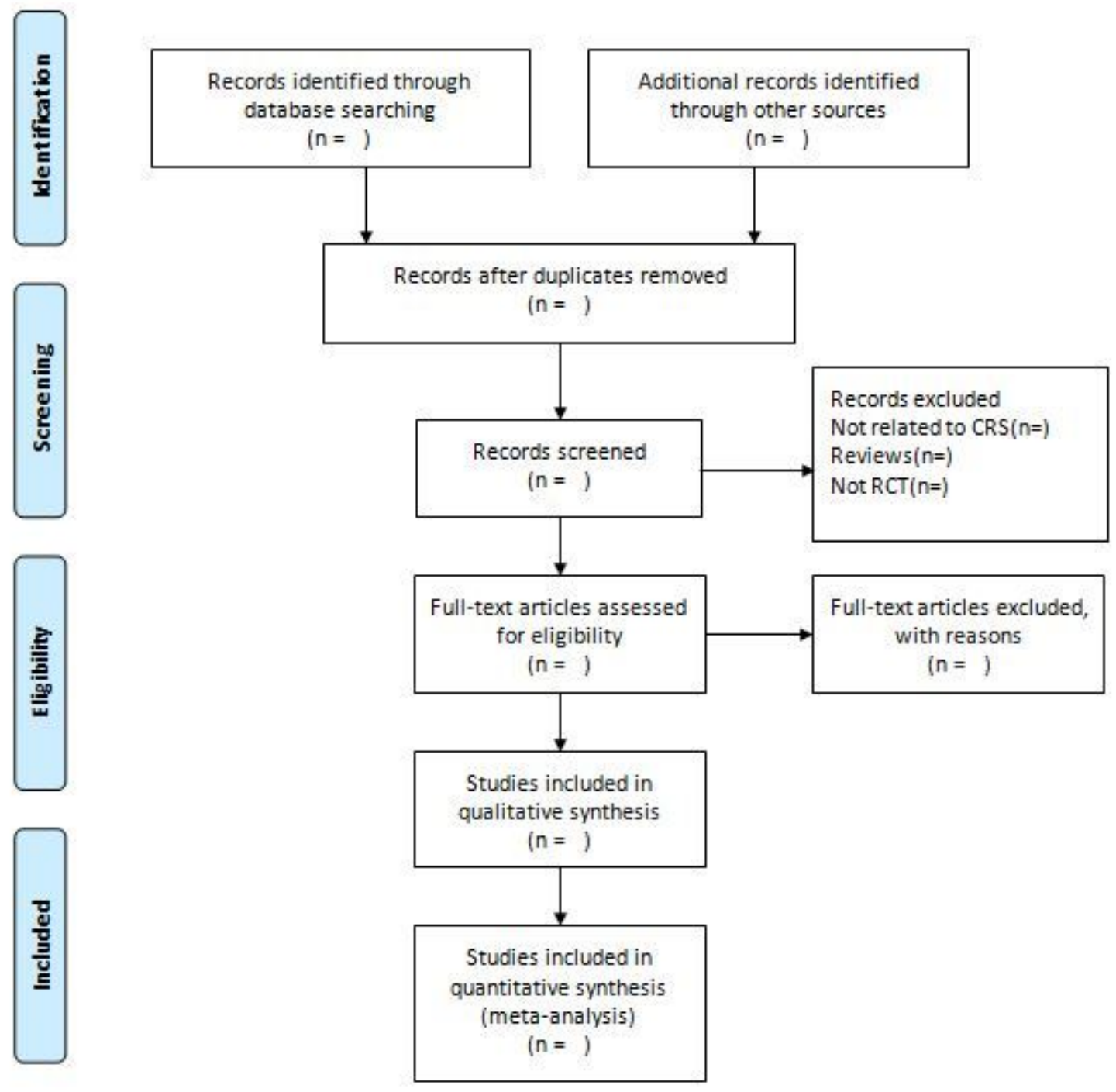

\section{Figure 1}

Search flow chart

\section{Supplementary Files}

This is a list of supplementary files associated with this preprint. Click to download.

- Additionalfile2.SearchStrategyforPubMed.docx

- Additionalfile1.PRISMAP2015Checklist.docx 